\title{
GENERATION OF TUNABLE INFRARED FEMTOSECOND PULSES VIA PARAMETRIC VISIBLE-TO-INFRARED FREQUENCY CONVERSION
}

\author{
J. Darginavičius 国, E. Rubino 日, G. Tamošauskas 国, D. Faccio 9 , G. Valiulis \\ P. Di Trapani ${ }^{\mathrm{b}}$, A. Piskarskas 3 , and A. Dubietis \\ ${ }^{a}$ Department of Quantum Electronics, Vilnius University, Saulètekio 9 bldg. 3, LT-10222 Vilnius, Lithuania \\ E-mail: audrius.dubietis@ ff.vu.lt \\ ${ }^{\mathrm{b}}$ CNISM and Department of Physics and Mathematics, Università dell 'Insubria, Via Vallegio 11, IT-22100 Como, Italy \\ ${ }^{c}$ School of Engineering and Physical Sciences, SUPA, Heriot-Watt University, Edinburgh EH14 4AS, UK
}

Received 23 May 2011; revised 31 August 2011; accepted 21 September 2011

\begin{abstract}
We propose two methods for ultrashort pulse parametric visible-to-infrared frequency conversion, that are directly applied to extend the tuning range of a commercial Ti:sapphire laser-noncollinear optical parametric amplifier system. The first method is based on broadband noncollinear four-wave optical parametric amplification in fused silica, and the second is based on cascaded three-wave parametric interaction in a single BBO crystal. The proof-of-principle experiments demonstrate generation of sub$30-\mathrm{fs}, \sim 20 \mu \mathrm{J}$ pulses, broadly tunable across the infrared $(1-3 \mu \mathrm{m})$ spectral range.
\end{abstract}

Keywords: optical parametric amplifiers, femtosecond pulses, infrared, four-wave mixing, difference frequency generation

PACS: $42.65 . \mathrm{Re}$, 42.65.Yj

\section{Introduction}

Optical parametric frequency conversion offers simple, reliable, and flexible method for generation of tunable intense femtosecond pulses; optical parametric amplifiers endow a broad wavelength tunability to any existing ultrashort pulsed laser system [1]. A considerable progress in widely tunable femtosecond pulse generation is closely related to the development of commercial amplified femtosecond Ti:sapphire laser systems, which nowadays provide stable, high energy and high peak power pulses with $30-150$ fs duration at fundamental $(800 \mathrm{~nm})$ and second harmonics $(400 \mathrm{~nm})$ wavelengths that readily serve for pumping of optical parametric frequency converters based on birefringent crystals with second-order nonlinearity. Ti:sapphire laser pumped optical parametric amplifiers based on beta-barium borate, $\mathrm{BaB}_{2} \mathrm{O}_{4}$ (BBO) crystal routinely provide femtosecond pulses tunable in the visible and near-infrared (IR) spectral range, see [2] for a review. However, generation and amplification of even shorter ( $<30 \mathrm{fs}$ ) pulses requires broad amplification bandwidth that is achieved by matching the group velocities of the signal and idler waves over fairly broad wavelength range by the use of noncollinear phase matching geome- try. This finding led to development of the noncollinear optical parametric amplification technique [3], which lies on the basis of the most advanced broadband optical parametric amplifiers.

Nowadays blue-pumped BBO crystal-based noncollinear optical parametric amplifiers (NOPAs) efficiently operate in the visible spectral range and serve as an indispensable tool for generation of ultrashort pulses with duration $<30$ fs [4]. However, in the light of success of blue-pumped NOPAs, generation of sub-30 fs pulses in the near IR is still a challenging task. This is because of BBO crystal-based NOPAs fail to support broadband phase matching for IR wavelengths using an $800 \mathrm{~nm}$ Ti:sapphire laser pump. Although the idler wave, which lies in the IR spectral range, is readily produced by the amplification process, its use is almost impractical because of irregular spatial chirp [5]. In this regard, the noncollinear optical parametric amplification with $800 \mathrm{~nm}$ pumping has been demonstrated using different nonlinear crystals, which provide suitable group velocity matching for broadband amplification: potassium titanyl phosphate (KTP) [6], bismuth triborate (BIBO) [7], lithium iodate [8], and periodically poled stoichiometric lithium tantalate (PPSLT) [9]; see 
also [10] for a review and recent developments in the field.

The existing alternatives for ultrashort pulse generation in the IR spectral range suggest optical parametric amplification in gases by means of four-wave parametric processes in the filamentation [11] and guidedwave propagation regimes [12] taking an advantage of intrinsically low group velocity dispersion of gaseous media and small group velocity mismatch between the signal and idler pulses. The latter guarantees broad amplification bandwidth that, when combined with nonlinear propagation effects, is able of supporting generation of IR pulses close to a single optical cycle limit [13]. Another approach considers IR generation in wide bandgap solids, such as $\mathrm{CaF}_{2}$ and $\mathrm{BaF}_{2}$ [14, 15], which possess much higher third-order nonlinearity than gases. A suitably chosen noncollinear interaction geometry allows to achieve high conversion efficiency (up to 10-15\%), high output energy, and broad amplification bandwidth [16-18]. Cascaded four-wave mixing offers additional possibilities for generation of tunable broadband multicolor output pulses with excellent self-compressibility and high temporal contrast [19].

An important feature of four-wave parametric interaction is that it enables to amplify and/or generate pulses with wavelength shorter than the pump, that is not possible with conventional three-wave optical parametric amplifiers, where wavelengths of both signal and idler waves are longer than the pump. This offers an additional degree of freedom in frequency conversion. Indeed, by taking an advantage of this possibility, generation of UV laser harmonics by means of four-wave difference frequency mixing [20] and UV-tunable 30-fs pulses by parametric frequency up-conversion of visible pulses [21] was demonstrated. More recently, a simi- lar technique was applied in the near-IR spectral range, yielding broadly tunable sub-30 fs pulses from fused silica based four-wave optical parametric amplifier [22].

In this paper we experimentally demonstrate efficient visible-to-infrared frequency conversion of the ultrashort light pulses for extension of the tuning range of a commercial Ti:sapphire laser-NOPA system. The laser system used in the experiments consists of a commercial amplified Ti:sapphire laser (Spitfire PRO, Newport-Spectra Physics) that provides 130 fs pulses with $800 \mathrm{~nm}$ central wavelength at $1 \mathrm{kHz}$ repetition rate and a commercial second-harmonic-pumped BBO crystal-based noncollinear parametric amplifier (Topaswhite, Light Conversion Ltd.) that produces $\sim 30$-fs pulses tunable in the visible $(550-700 \mathrm{~nm})$ spectral range.

\section{Generation of sub-30 fs pulses by noncollinear optical parametric amplification in fused silica}

In this Section we propose a method, how broadband visible pulses delivered from a commercial Ti:sapphireNOPA system could be efficiently converted into the near-IR spectral range, utilizing single-pass four-wave optical parametric amplification in fused silica sample and subsequent compression of the frequency upconverted (idler) pulses.

The experimental set-up is depicted in Fig. 1. The laser beam at the fundamental frequency was divided by $30 \%$ beam splitter BS into two parts: one part (with energy variable up to $1.8 \mathrm{~mJ}$ ) served as a pump for the four-wave optical parametric amplifier (FWOPA), while the second part (with energy $0.5 \mathrm{~mJ}$ ) was frequency doubled in 1-mm-thick BBO crystal (SHG) and served to pump a NOPA device, which provided a broadband seed signal with a duration of $30 \mathrm{fs}$ and

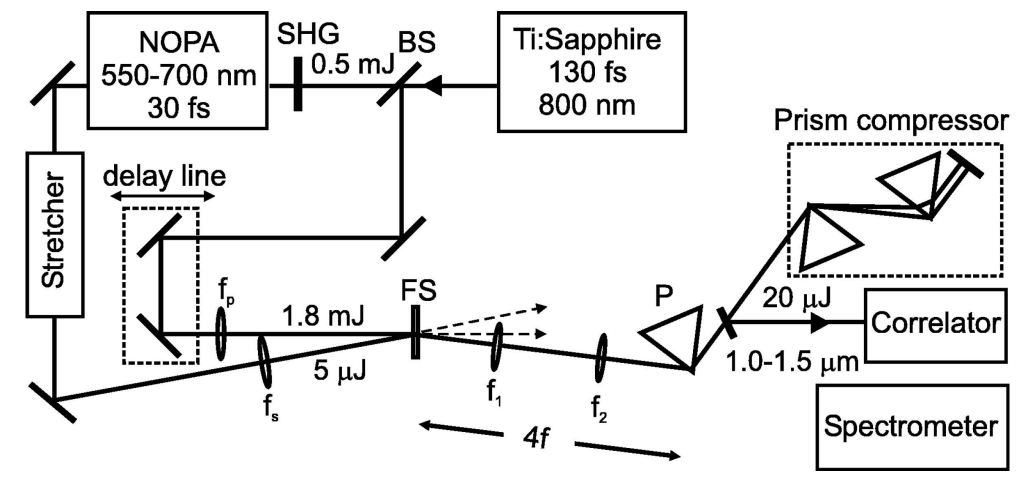

Fig. 1. Experimental set-up for four-wave optical parametric amplification: $B S$ is the beamsplitter, $S H G$ is the second-harmonic generating crystal, $F S$ is the fused silica plate, $P$ is the pulse-front tilt compensating prism, $f_{\mathrm{p}}$ and $f_{\mathrm{s}}$ are the focusing and $f_{1}$ and $f_{2}$ are the imaging lenses, respectively. 
which was tunable in the visible wavelength range $(550-700 \mathrm{~nm})$. The seed signal pulse was additionally stretched from 30 to 50 fs by passing through 7-mmlong slab of silica glass, denoted as a pulse stretcher in Fig. 1, hence acquiring a positive chirp. The pump and seed signal beams of the same linear polarization were noncollinearly overlapped at the phase-matching angle inside the 1-mm-long fused-silica sample (FS). The pump and seed signal beams were focused with spherical lenses, $f_{\mathrm{p}}=800 \mathrm{~mm}$ and $f_{\mathrm{s}}=500 \mathrm{~mm}$, respectively, that yielded respective FWHM beam dimensions of 0.8 and $0.33 \mathrm{~mm}$ at the input facet of the fused silica sample, ensuring a good spatial overlap of the interacting waves along the sample length.

The phase-matching angle between the pump and signal beams, which supports efficient four-wave parametric interaction $\left(\omega_{\mathrm{p}}+\omega_{\mathrm{p}}=\omega_{\mathrm{s}}+\omega_{\mathrm{i}}\right)$, was calculated according to equation [23]:

$$
\cos \theta_{\mathrm{s}}=\frac{4 k_{\mathrm{p}}^{2}+k_{\mathrm{s}}^{2}-k_{\mathrm{i}}^{2}}{4 k_{\mathrm{s}} k_{\mathrm{p}}},
$$

where $k(\omega)=n(\omega) \omega / c$ is the wave number, $n(\omega)$ is the refractive index, and subscripts $\mathrm{p}, \mathrm{s}$, and i refer to pump, signal, and idler (near-IR) waves, respectively. The angle between the pump and emerging idler wave was therefore found as

$$
\cos \theta_{\mathrm{i}}=\frac{4 k_{\mathrm{p}}^{2}+k_{\mathrm{i}}^{2}-k_{\mathrm{s}}^{2}}{4 k_{\mathrm{i}} k_{\mathrm{p}}} .
$$
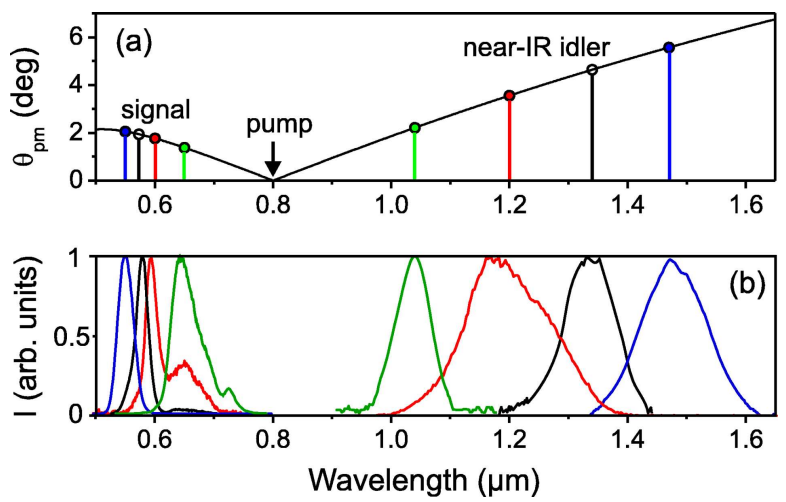

Fig. 2. (a) Phase-matching curve in fused silica. (b) Spectra of the amplified signal and generated near-IR (idler) pulses.

The phase-matching curve in fused silica is depicted in Fig. 2(a), showing that phase-matching angles for a visible seed signal vary between 1.5 and 2.1 degrees, whereas those for the idler (near-IR) pulses vary in the range of $2 \cdot 2-5 \cdot 5^{\circ}$. Figure 2 (b) shows typical spectra of the amplified signal and generated near-IR pulses. With a NOPA signal tuning range of $550-700 \mathrm{~nm}$, the generated near-IR idler pulses cover wavelength range from 1.0 to $1.5 \mu \mathrm{m}$. The near-IR pulse spectrum was recorded by a fibre spectrometer $(Q E 65000$, Ocean Optics) after the frequency doubling in thin, $20-\mu \mathrm{m} \mathrm{BBO}$ crystal that ensured broadband frequency conversion from IR to visible so as to fit the detection range of the spectrometer. The measured spectral FWHM width of the near-IR pulses varied from 70 to $140 \mathrm{~nm}$, supporting short, sub-30-fs pulse duration throughout the whole tuning range and even sub-20-fs duration for the near-IR pulse centred at $1.2 \mu \mathrm{m}$, where NOPA provides the broadest spectra.
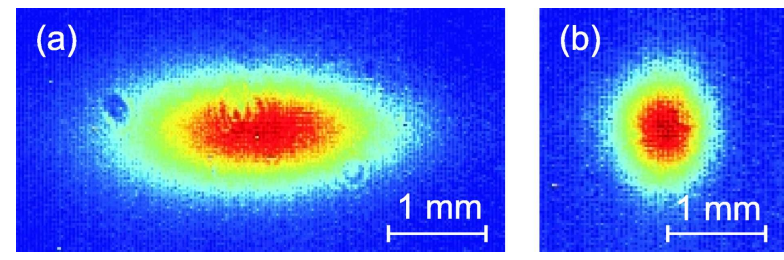

Fig. 3. Far-field intensity profiles of the near-IR beam (at $1.34 \mu \mathrm{m}$ ): (a) before and (b) after the pulse-front tilt removal.

Due to noncollinear interaction geometry, the generated broadband near-IR pulse possessed a pulse-front tilt, which manifested itself as apparent angular dispersion of the beam in the phase-matching plane, that is clearly visible as elongated far-field intensity profile of the near-IR beam, as shown in Fig. 3(a). The pulse-front tilt was fairly constant along the whole tuning range as a result of the constant slope of the phase-matching curve. The tilt value was evaluated as $\delta=\arctan (\lambda \mathrm{d} \theta / \mathrm{d} \lambda) \approx$ $9.9^{\circ}$. The pulse-front tilt was removed by imaging the near-IR pulse with a beam-size magnifying telescope consisting of $f_{1}=125 \mathrm{~mm}$ and $f_{2}=300 \mathrm{~mm}$ lenses, arranged in $4 f$ geometry, onto a single TF10 glass prism $(\mathrm{P})$ with a $60^{\circ}$ apex angle. Figure 3 compares the farfield intensity profiles of the near-IR beam before and after the tilt removal, as recorded by InGaAs complementary metal-oxide semiconductor (CMOS) camera (Xenics, Xeva 202). After the tilt removal, the pulse was compressed in a double-pass prism-pair compressor that consisted of two identical $60^{\circ}$ apex angle TF5 glass prisms aligned at the angle of minimum deviation.

Figure 4 illustrates the relevant characteristics of the FWOPA operation at two wavelengths, $1.34 \mu \mathrm{m}$ and $1.20 \mu \mathrm{m}$. The left panel (Fig. $4(\mathrm{a}, \mathrm{d})$ ) compares spectra of the seed and amplified signal in the visible range. The centre panel (Fig. $4(\mathrm{~b}, \mathrm{e})$ ) illustrates spectra of the frequency down-converted near-IR pulses at the FWOPA output. The right panel (Fig. 4(c, f)) shows the autocorrelation functions of the compressed nearIR pulses as measured by a scanning autocorrelator. 

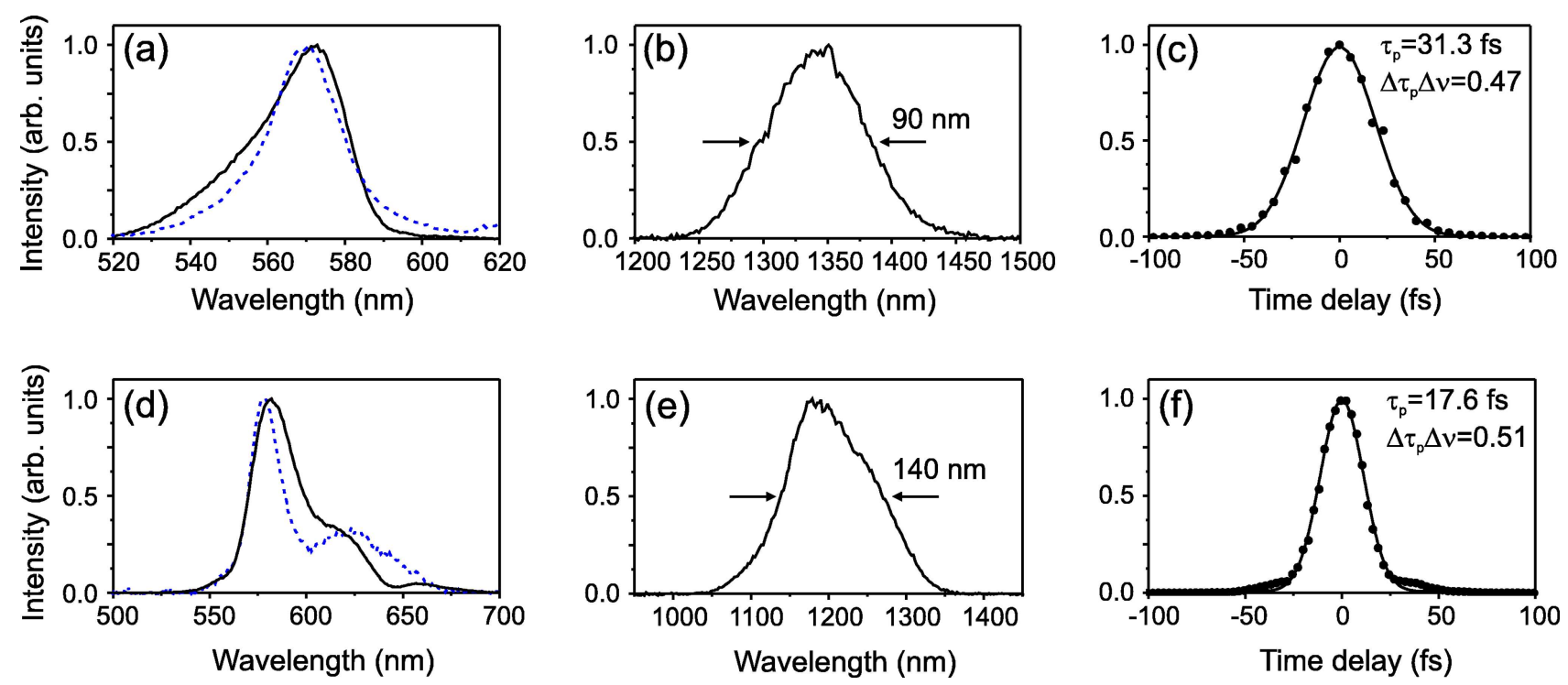

Fig. 4. Pulse characteristics at the FWOPA output at $1.34 \mu \mathrm{m}$ (upper row) and $1.20 \mu \mathrm{m}$ (lower row): (a), (d) spectra of the seed (dashed curves) and amplified signal (solid curves) in the visible; (b), (e) spectra of the near-IR pulses; (c), (f) autocorrelation traces of compressed near-IR pulses. Here solid curves show the Gaussian fit.

The estimated time-bandwidth product $\Delta \tau_{\mathrm{p}} \Delta \nu \approx 0.5$ at both wavelengths indicates that the near-IR pulses are well compressible to almost bandwidth limit. It is worth noting that the time-bandwidth product of the near-IR pulses was fairly constant across the entire tuning range $(1-1.5 \mu \mathrm{m})$. The shortest near-IR pulse of 17.6 fs duration that equates to 4.4 optical cycles, was measured at $1.2 \mu \mathrm{m}$, as shown in Fig. $4(\mathrm{f})$.

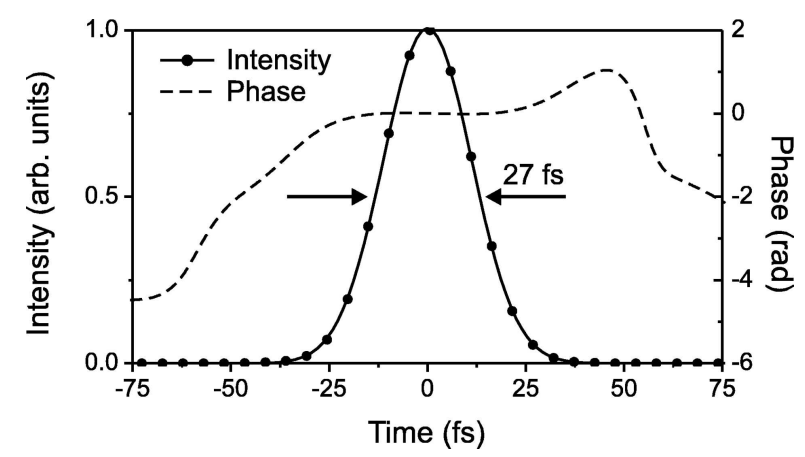

Fig. 5. SHG-FROG characterization of the near-IR pulse at $1.34 \mu \mathrm{m}$, showing retrieved temporal intensity (solid curve) and phase (dashed curve) profiles.

We also observed that an increase of the pump energy leads to a spectral broadening of the near-IR pulse, as a result of the cross-phase modulation (XPM) between the pump and seed pulses inside the fused silica sample, as noted in similar experiments [18, 24]. We have found that the XPM-broadened pulses are still fully compressible, and indeed, the measured pulse durations, after optimization of the pulse compressor, have been shorter by $\sim 10-20 \%$. An example of the XPM-broadened pulse compression is illustrated in Fig. 5 that depicts temporal intensity and phase profiles of the compressed pulse at $1.34 \mu \mathrm{m}$ generated with a $1.8 \mathrm{~mJ}$ pump, as retrieved by SHG-FROG (Second Harmonic Generation Frequency Resolved Optical Gating) technique.

Using a seed signal energy of $4.8 \mu \mathrm{J}$, which was kept fairly constant across the whole visible spectral range, and pump energy of $1.8 \mathrm{~mJ}$, FWOPA delivered sub30 -fs near-IR pulses with energy up to $20 \mu \mathrm{J}$ across the whole $1-1.5 \mu \mathrm{m}$ wavelength range. Under these settings, $1 \%$ pump-to-idler conversion efficiency was achieved, that is competitive to frequency conversion schemes based on gaseous media. Notably, the conversion efficiency could be further increased either by optimizing (increasing) the input seed energy or by better matching of the pump and seed pulsewidths.

\section{Generation of broadly tunable ultrashort IR pulses by cascaded frequency mixing in a single BBO crystal}

The noncollinear geometry of the four-wave optical parametric amplification might be somewhat less technically attractive if fast wavelength tunability (scanning) is required, since the wavelength tuning requires adjustment of the beam intersection angle so as to fulfil the phase matching condition, as seen from Fig. 2(a). Interestingly, wavelength tuning could also be performed at a fixed angle, by choosing a set of various nonlinear media [25]. In this Section we demonstrate a different approach for generation of broadly tunable ul- 


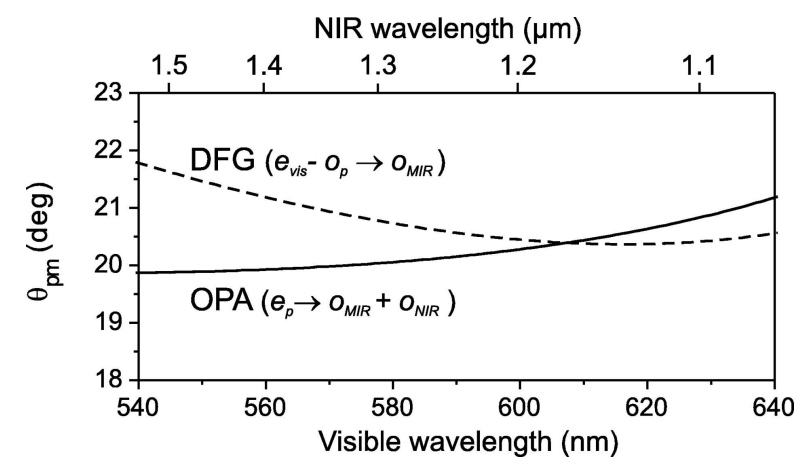

Fig. 6. Phase matching curves for DFG (dashed line) and OPA (solid line) processes for type I phase matching in BBO crystal.

trashort near-IR pulses by means of collinear cascaded three-wave parametric interactions in a single BBO crystal. The idea of the method is that tunable IR pulses are generated by means of cascaded second-order parametric interactions: difference-frequency generation (DFG) and optical parametric amplification (OPA) that occur simultaneously within a single nonlinear crystal.

Schematically, the whole frequency conversion process could be viewed as a two-step three-wave interaction. The first step is the difference-frequency generation via parametric process between a visible pulse delivered from NOPA, $\omega_{\text {vis }}$, and a Ti:sapphire fundamental harmonics pulse (centre wavelength $800 \mathrm{~nm}, \omega_{\mathrm{p}}$ ) thus producing the mid-IR pulse at $\omega_{\mathrm{MIR}}=\omega_{\mathrm{vis}}-\omega_{\mathrm{p}}$. The second step is the optical parametric amplification of the difference-frequency (mid-IR) wave, which serves as a seed signal, at the same time producing the near-IR idler wave according to energy conservation: $\omega_{\mathrm{p}}=\omega_{\mathrm{MIR}}+\omega_{\mathrm{NIR}}$. Specifically, by tuning the wavelength of the visible pulse in the $\lambda_{\text {vis }}=560-630 \mathrm{~nm}$ wavelength range, we obtain simultaneous generation and amplification of broadly tunable IR pulses within the wavelength range of $\lambda_{\mathrm{MIR}}=1.9-3 \mu \mathrm{m}$ and $\lambda_{\mathrm{NIR}}=$ $1.1-1.4 \mu \mathrm{m}$, respectively.
The efficiency of the overall cascading process relies on two conditions: (i) both parametric interactions have to be of the same phase matching type, thus the polarization of the interacting waves should be properly chosen; (ii) only one of the two parametric processes could be perfectly phase matched at a fixed crystal orientation. This is illustrated in Fig. 6 by plotting the phase-matching curves in BBO crystal for both DFG $\left(o_{\mathrm{MIR}} \rightarrow e_{\mathrm{vis}}-o_{\mathrm{p}}\right)$ and OPA $\left(e_{\mathrm{p}} \rightarrow o_{\mathrm{MIR}}+o_{\mathrm{NIR}}\right)$ processes. Here $o$ and $e$ denote the necessary polarization of the interacting waves so as to fulfil the type I phase matching interaction. Note that this is possible using different polarizations of the pump wave at $\omega_{\mathrm{p}}$. The optimum operation was achieved when the BBO crystal was aligned so as to fulfil the perfect phase matching condition for the OPA process that is very efficient, whereas a difference frequency generation was phasemismatched thereby providing a weak seed, which was nevertheless enough to efficiently seed the amplifier. The tuning range of the mid-IR pulses and therefore of the whole set-up was limited by the IR absorption in the BBO crystal.

The experimental set-up is depicted in Fig. 7. The Ti:sapphire laser-NOPA system was the same as described in the previous section. A dichroic mirror DM (transparent in the visible and highly reflective at $800 \mathrm{~nm}$ ) was used for collinear arrangement of the NOPA and pump beams. The two beams were focused with a spherical $f_{1}=+800 \mathrm{~mm}$ lens into a 1 -mm-thick $\mathrm{BBO}$ crystal cut at $\theta=29.2^{\circ}$. The BBO crystal was placed $\sim 20 \mathrm{~cm}$ before the focal plane of the lens; the measured FWHM size of pump and signal beams at the input face of the crystal were 0.77 and $0.45 \mathrm{~mm}$, respectively. The generated near-IR beam was restored with an $f_{2}=500 \mathrm{~mm}$ lens, while the remaining pump and NOPA signal were filtered using a bandpass filter $\mathrm{F}$. The proper energy ratio between $o$ - and $e$-polarized

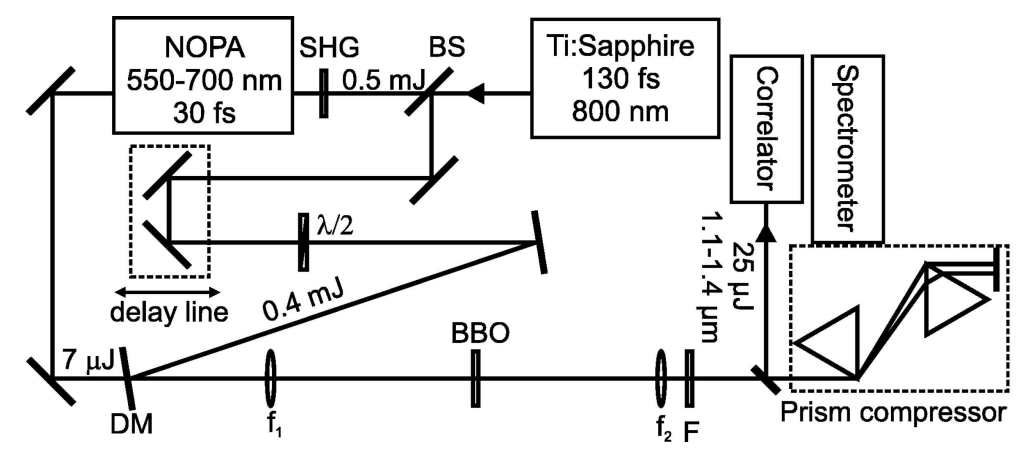

Fig. 7. Experimental set-up for cascaded frequency conversion in BBO crystal: BS is the beamsplitter, SHG is the second-harmonic generating crystal, $\lambda / 2$ is a half-wave plate, DM is a dichroic mirror, $f_{1}$ and $f_{2}$ are beam focusing and restoring lenses, respectively, $\mathrm{F}$ is a bandpass filter. 
waves at $800 \mathrm{~nm}$ was adjusted with a half-wave plate $(\lambda / 2)$. The optimum performance was achieved when $\sim 5 \%$ fraction of the energy at $800 \mathrm{~nm}$ was used to generate the difference-frequency and the rest $\sim 95 \%$ served as a pump for OPA. The maximum pump energy of $E_{\mathrm{p}}=0.4 \mathrm{~mJ}$ in the experiment was used, and that converts to a maximum fluence $F_{\max }$ of $0.056 \mathrm{~J} / \mathrm{cm}^{2}$. We note that no optical damage of the crystal antireflection coatings was observed. The maximum pump intensity was estimated as $I_{\max }=0.43 \mathrm{TW} / \mathrm{cm}^{2}$, assuming Gaussian spatial and temporal intensity distribution of the pump beam.
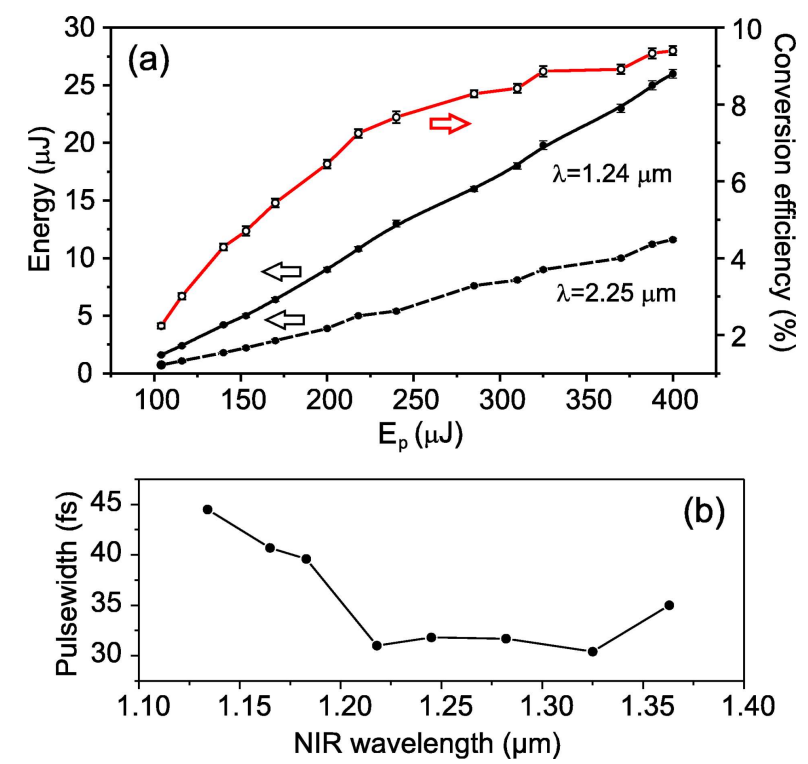

Fig. 8. (a) Infrared pulse energies at $2.25 \mu \mathrm{m}$ (dashed curve) and $1.24 \mu \mathrm{m}$ (solid curve) and pump-to-infrared conversion efficiency versus pump energy. (b) Compressed near-IR pulse duration versus wavelength.

Figure 8 (a) shows the energy of the near-IR (at $1.24 \mu \mathrm{m}$ ) and mid-IR (at $2.25 \mu \mathrm{m}$ ) pulses at the BBO crystal output as a function of the pump energy. With $E_{\mathrm{p}}=0.4 \mathrm{~mJ}$ and $E_{\mathrm{vis}}=7 \mu \mathrm{J}$ the highest infrared pulse energies $E_{\mathrm{MIR}}=11.5 \mu \mathrm{J}$ and $E_{\mathrm{NIR}}=26 \mu \mathrm{J}$ were measured suggesting almost $10 \%$ pump-to-infrared conversion efficiency as the frequency conversion process was driven into saturation. Figure 8(b) shows the near-IR pulse duration versus wavelength after the pulse compression in a double-pass prism-pair compressor (identical to that described in Sec. 2) as measured by a scanning autocorrelator. For most of the tuning range, pulses as short as $30 \mathrm{fs}$ were obtained, whereas at the shortest wavelengths $(1.1-1.2 \mu \mathrm{m})$, the pulse duration increased to 40-45 fs, mainly as a result of reduced gain bandwidth for shortest near-IR wavelengths.
Figure 9 summarizes the relevant characteristics of the cascaded three-wave parametric frequency converter at 1.36 and $1.22 \mu \mathrm{m}$. The left panel (Fig. $9(\mathrm{a}, \mathrm{d})$ ) compares spectra of the visible NOPA seed. The centre panel (Fig. 9(b,e)) illustrates spectra of the frequency down-converted near-IR pulses. The right panel (Fig. $9(\mathrm{c}, \mathrm{f}))$ shows the autocorrelation traces of the compressed near-IR pulses as measured by a scanning autocorrelator. The estimated time-bandwidth product $\Delta \tau_{\mathrm{p}} \Delta \nu \approx 0.5$ at both wavelengths indicates that the compressed near-IR pulses are close to transform limit.

In fact, such second-order cascaded interaction to some extent mimics pure four-wave mixing process: $\omega_{\mathrm{p}}+\omega_{\mathrm{p}}=\omega_{\mathrm{vis}}+\omega_{\mathrm{NIR}}$. However, in the present case the conversion efficiency is markedly higher because of much higher induced second-order nonlinear polarization that is the source of oscillation at new frequencies. Another important outcome of the proposed scheme is that tunable mid-IR pulses via DFG process are simultaneously generated, and those are not present in pure four-wave mixing process. In our experiment, these pulses were not precisely characterized due to absence of suitable (mid-IR sensitive) detection apparatus. However, it is worth mentioning that in the DFG process, the difference frequency pulse emerges with a stable carrier envelope phase offset if the pump and the seed pulses are derived from the same source [26], and this is exactly the case. Moreover, the proposed method is suitable for visible-to-mid-IR conversion (for example, visible pulses in the wavelength range of 630$700 \mathrm{~nm}$ could be converted to a wavelength range of $3-$ $5.6 \mu \mathrm{m}$ in the mid-IR), using nonlinear crystals which are transparent in the mid-IR.

\section{Conclusions}

To summarize the results, we have demonstrated two different approaches for tunable ultrashort $(\leq 30 \mathrm{fs}$ ) pulse generation in the IR spectral range by visible-toinfrared frequency conversion. In contrast to widely used schemes that involve optical parametric amplification of the IR part of the white light continuum spectrum [10], the proposed schemes benefit from using already well-established ultrashort visible light pulses, which are of excellent spatial and temporal quality and high energy.

The first demonstrated approach relies on the fourwave parametric amplification, which to the best of our knowledge, is the first scheme based on isotropic solidstate medium (fused silica) that allows visible-to-IR conversion and provides both very large tunability and 

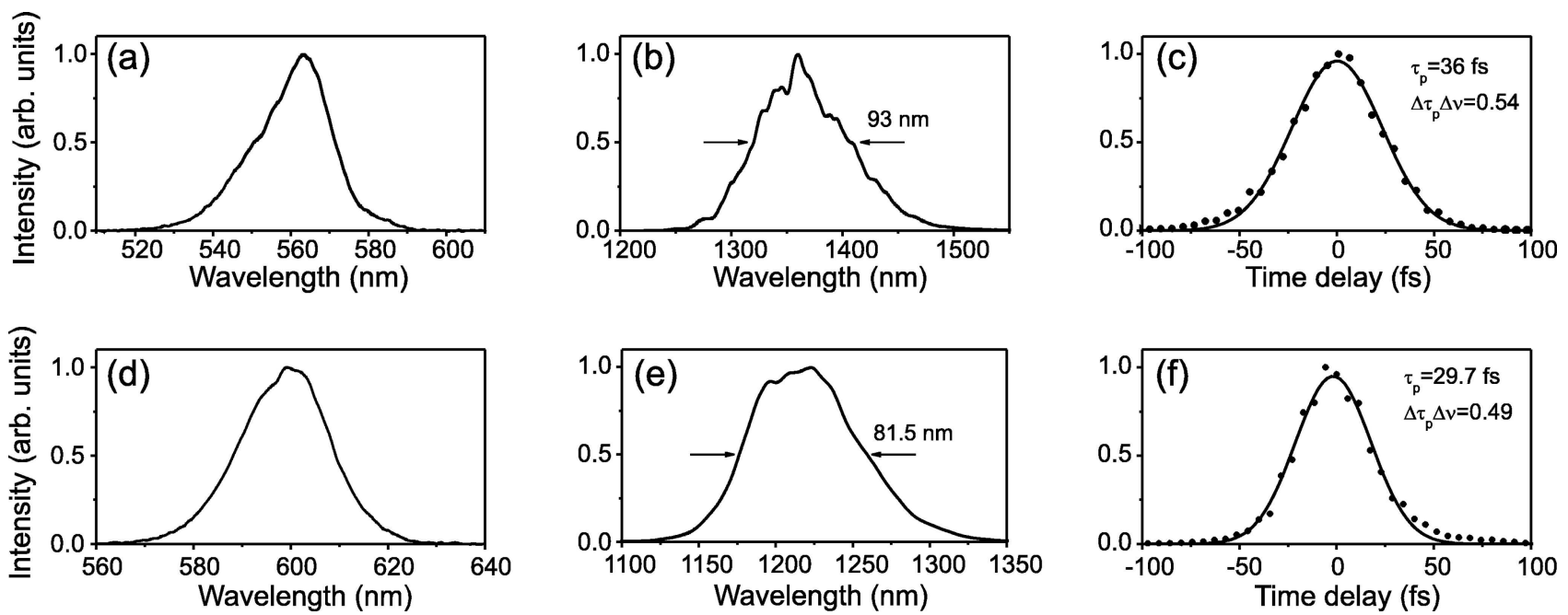

Fig. 9. Experimentally measured pulse characteristics at $1.36 \mu \mathrm{m}$ (upper row) and $1.22 \mu \mathrm{m}$ (lower row): (a), (d) spectra of the visible NOPA seed; (b), (e) spectra of the near-IR pulses; (c), (f) autocorrelation traces of compressed near-IR pulses. Here solid curves show the Gaussian fit.

simultaneous broadband amplification, supporting fewoptical-cycle pulses in the near-IR $(1-1.5 \mu \mathrm{m})$ spectral range. This approach promises the scaling to very high pump energy levels due to the availability of large aperture media with Kerr nonlinearity and suitable phasematching conditions for frequency down-conversion further into IR, using materials with extremely broadband transparency range, such as $\mathrm{CaF}_{2}$.

The second demonstrated approach is based on cascaded three-wave interactions in a single BBO crystal, yielding difference frequency generation and optical parametric amplification. The major advantage of this scheme is the collinear interaction geometry, which allows easy and fast wavelength tuning and at the same time demonstrates high parametric gain and supports sufficiently broad amplification bandwidth. The midIR pulses generated in the wavelength range of 1.9$3.0 \mu \mathrm{m}$ should have stable carrier envelope phase offset, which is an important attribute of few-optical cycle pulses required for many applications in controlled light and matter interactions. By this technique, using the nonlinear crystals with better IR transparency, it is possible to obtain tunable pulses in the mid-IR $(3-5.6 \mu \mathrm{m})$ with tens of $\mu \mathrm{J}$ energy and with stable carrier envelope phase offset.

The developed IR sources might readily serve for diverse spectroscopic applications, and they might be conveniently used as energetic seed pulses with a high spatial and temporal quality for front-end high-power OPCPA based laser systems operating in the IR [27].

\section{Acknowledgements}

JD, GT, GV and AD acknowledge the financial support from the Research Council of Lithuania (project FORTAS, AUT-04/2010). ER and DF had received funding from the EC's Seventh Framework Programme (LASERLAB-EUROPE access, grant agreement No. 228334).

\section{References}

[1] R. Danielius, A. Piskarskas, A. Stabinis, G.P. Banfi, P. Di Trapani, and R. Righini, Traveling-wave parametric generation of widely tunable, highly coherent femtosecond light pulses, J. Opt. Soc. Am. B 10, 22222232 (1993).

[2] G. Cerullo and S. De Silvestri, Ultrafast optical parametric amplifiers, Rev. Sci. Instrum. 74, 1-18 (2003).

[3] T. Wilhelm, J. Piel, and E. Riedle, Sub-20-fs pulses tunable across the visible from a blue-pumped single-pass noncollinear parametric converter, Opt. Lett. 22, 14941496 (1997).

[4] E. Riedle, M. Beutter, S. Lochbrunner, J. Piel, S. Schenkl, S. Spörlein, and W. Zinth, Generation of 10 to $50 \mathrm{fs}$ pulses tunable through all of the visible and NIR, Appl. Phys. B 71, 457-465 (2000).

[5] A. Shirakawa, I. Sakane, and T. Kobayashi, Pulsefront-matched optical parametric amplification for sub10 -fs pulse generation tunable in the visible and near infrared, Opt. Lett. 23, 1292-1294 (1998).

[6] O. Isaienko and E. Borguet, Genereation of ultrabroadband pulses in the near-IR by noncollinear optical parametric amlification in potassium titanyl phosphate, Opt. Express 16, 3949-3954 (2008).

[7] M. Ghotbi, V. Petrov, and F. Noack, Broadly tunable, sub-30 fs near-infrared pulses from an optical paramet- 
ric amplifier based on $\mathrm{BiB}_{3} \mathrm{O}_{6}$, Opt. Lett. 35, 21392141 (2010).

[8] D. Brida, C. Manzoni, G. Cirmi, M. Marangomi, S. De Silvestri, and G. Cerullo, Generation of broadband mid-infrered pulses from an optical parametric amplifier, Opt. Express 15, 15035-15040 (2007).

[9] D. Brida, S. Bonora, C. Manzoni, M. Marangoni, P. Villoresi, S. De Silvestri, and G. Cerullo, Generation of 8.5 -fs pulses at $1.3 \mu \mathrm{m}$ for ultrabroadband pumpprobe spectroscopy, Opt. Express 17, 12510-12515 (2009).

[10] D. Brida, C. Manzoni, G. Cirmi, M. Marangoni, S. Bonora, P. Villoresi, S. De Silvestri, and G. Cerullo, Few-optical cycle pulses tunable from the visible to the mid-infrared by optical parametric amplifiers, J. Opt. A 12, 013001 (2010).

[11] T. Fuji and T. Suzuki, Generation of sub-two-cycle mid-infrared pulses by four-wave mixing through filamentation in air, Opt. Lett. 32, 3330-3332 (2007).

[12] D. Faccio, A. Grün, P.K. Bates, O. Chalus, and J. Biegert, Optical amplification in the near-infrared in gas-filled hollow-core fibers, Opt. Lett. 34, 2918-2920 (2009).

[13] L. Bergé, Self-compression of $2 \mu \mathrm{m}$ laser filaments, Opt. Express 16, 21529-21543 (2008).

[14] H. Okamoto and M. Tatsumi, Generation of ultrashort light pulses in the mid-infrared $\left(3000-800 \mathrm{~cm}^{-1}\right)$ by four-wave mixing, Opt. Commun. 121, 63-68 (1995).

[15] H.-K. Nienhuys, P.C.M. Planken, R.A. van Santen, and H.J. Bakker, Generation of mid-infrared pulses by $\chi^{(3)}$ difference frequency generation in $\mathrm{CaF}_{2}$ and $\mathrm{BaF}_{2}$, Opt. Lett. 26, 1350-1352 (2001).

[16] A. Dubietis, G. Tamošauskas, P. Polesana, G. Valiulis, H. Valtna, D. Faccio, P. Di Trapani, and A. Piskarskas, Highly efficient four-wave parametric amplification in transparent bulk Kerr medium, Opt. Express 15, 11126-11132 (2007).

[17] H. Valtna, G. Tamošauskas, A. Dubietis, and A. Piskarskas, High-energy broadband four-wave optical parametric amplification in bulk fused silica, Opt. Lett. 33, 971-973 (2008).

[18] J. Darginavičius, G. Tamošauskas, G. Valiulis, and A. Dubietis, Broadband four-wave optical parametric amplification in bulk isotropic media in the ultraviolet, Opt. Commun. 282, 2995-2999 (2009).

[19] T. Kobayashi, J. Liu, and Y. Kida, Generation and optimization of femtosecond pulses by four-wave mixing process, IEEE J. Sel. Top. Quantum Electron. [in press], doi:10.1109/JSTQE.2011.2105256.

[20] A. Dubietis, J. Darginavičius, G. Tamošauskas, G. Valiulis, and A. Piskarskas, Generation and amplification of ultrashort UV pulses via parametric four-wave interactions in transparent solid state media, Lith. J. Phys. 49, 421-431 (2009).

[21] J. Darginavičius, G. Tamošauskas, A. Piskarskas, and A. Dubietis, Generation of 30-fs ultraviolet pulses by four-wave optical parametric chirped pulse amplification, Opt. Express 18, 16096-16101 (2010).

[22] E. Rubino, J. Darginavičius, D. Faccio, P. Di Trapani, A. Piskarskas, and A. Dubietis, Generation of broadly tunable sub-30-fs infrared pulses by four-wave optical parametric amplification, Opt. Lett. 36, 382-384 (2011).

[23] A. Penzkofer and H.J. Lehmeier, Theoretical investigation of noncollinear phase-matched parametric fourphoton amplification of ultrashort light pulses in isotropic media, Opt. Quantum Electron. 25, 815-844 (1993).

[24] J. Liu, Y. Kida, T. Teramoto, and T. Kobayashi, Simultaneous compression and amplification of a laser pulse in a glass plate, Opt. Express 18, 2495-2502 (2010).

[25] J. Liu and T. Kobayashi, Cascaded four-wave mixing in transparent bulk media, Opt. Commun. 283, 11141123 (2010).

[26] A. Baltuška, T. Fuji, and T. Kobayashi, Controlling the carrier-envelope phase of ultrashort light pulses with optical parametric amplifiers, Phys. Rev. Lett. 88, 133901 (2002).

[27] O.D. Mücke, D. Sidorov, P. Dombi, A. Pugžlys, A. Baltuška, S. Ališauskas, V. Smilgevičius, J. Pocius, L. Giniūnas, R. Danielius, and N. Forget, Scalable YbMOPA-driven carrier envelope phase-stable few-cycle parametric amplifier at $1.5 \mu \mathrm{m}$, Opt. Lett. 34, 118-120 (2009). 


\section{DERINAMO DAŽNIO INFRARAUDONŲJŲ FEMTOSEKUNDINIŲ IMPULSŲ GENERAVIMAS PARAMETRIŠKAI KEIČIANT REGIMOSIOS SPINDULIUOTĖS DAŽNI}

J. Darginavičius ${ }^{\text {a }}$, E. Rubino ${ }^{\text {b }}$, G. Tamošauskas ${ }^{\text {a }}$, D. Faccio ${ }^{\text {c }}$, G. Valiulis ${ }^{\text {a }}$, P. Di Trapani ${ }^{\text {b }}$, A. Piskarskas ${ }^{\text {a }}$, A. Dubietis ${ }^{a}$

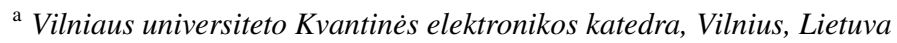

${ }^{\mathrm{b}}$ CNISM ir Insubrijos universiteto Fizikos ir matematikos katedra, Komo, Italija

${ }^{\mathrm{c}}$ SUPA ir Heriot-Watt universiteto Inžinerijos ir fiziniu mokslu mokykla, Edinburgas, Jungtinè Karalystè

\section{Santrauka}

Pasiūlyti du metodai parametriškai keisti regimają spinduliuotę infraraudonaja, leidžiantys praplèsti komercinès Ti:safyro lazerio ir nekolinearaus parametrinio šviesos stiprintuvo sistemos dažnių derinimo sriti. Pirmasis metodas remiasi plačiajuosčiu nekolineariu keturbangiu optiniu parametriniu stiprinimu lydytame kvarce, o antrasis - pakopinemis trijų bangų sąveikomis BBO kristale. Eksperimentiškai pademonstruotas mažesnès nei 30-fs trukmės $\sim 20 \mu \mathrm{J}$ energijos impulsų, derinamų infraraudonojoje spektro srityje (1$3 \mu \mathrm{m})$, generavimas. 